\title{
MS43-03 | Study of the Confinement Effect of Water in Bioactive Glasses Using
}

\section{ATOMIC PAIR}

Khoder, Hassan (Université de Lorraine, Vandoeuvres Les Nancy CEDEX, FRA); Bendeif, El-Eulmi (Université de Lorraine, Vandoeuvres Les Nancy CEDEX, FRA); Schaniel, Dominik (Université de Lorraine, Vandoeuvres Les Nancy CEDEX, FRA); DorbezSridi, Rachida (Université de Monastir, Monastir, TUN); Pillet, Sébastien (Université de Lorraine, Vandoeuvres Les Nancy CEDEX, FRA)

Significant therapeutical progress has been achieved in recent years by using bioactive glasses for bone reparation and replacement [1-4]. The biomedical applications of these biomaterials are mainly due to their high biocompatibility and high reactivity with the human physiological environment, since the reaction products obtained from these bioglasses and the physiological fluids lead to the deposition of a layer of crystalline bone-like carbonate calcium phosphate (Hydroxy-Carbonate Apatite, HCA) on their surface shortly after interaction. The internal porosity of these biomaterials further allows for progressive colonization of the tissues, ensures the vascularization and free circulation of cells, body fluids and nutriments.

The understanding of the impact of the confinement on the organization and diffusion of the physiological liquids encapsulated in these bio-nanomaterials is thus crucial for improving the bioglasses properties. We will present in this contribution, the detailed structural analysis of confined physiological liquids inside new mesoporous bioactive glasses. The structural investigations were performed using a complementary approach based on total X-ray scattering data coupled with the PDF analysis and appropriate calorimetric (DSC) measurements. Furthermore, we will discuss the comparison of the experimental and the simulated PDFs parameters.

[1] Hench, L. L. Science. 1984, 226, 630.

[2] Hench, L. L.; Polak, J. M. Science 2002, 295, 1014.

[3] Hench, L. L. J. Am. Ceram. Soc. 1998, 81, 1705.

[4] Izquierdo-Barba, I.; Arcos, D.; Sakamoto, Y.; Terasaki, O.; López-Noriega, A.; Vallet-Regí, M. Chemistry of Materials 2008, 20 (9), 3191-3198 